\title{
Estimating Progression Rates Across the Spectrum of Alzheimer's Disease for Amyloid-Positive Individuals Using National Alzheimer's Coordinating Center Data
}

\author{
Michele Potashman • Marric Buessing • Mihaela Levitchi Benea • \\ Jeffrey Cummings · Soo Borson · Peter Pemberton-Ross • \\ Andrew J. Epstein
}

Received: June 17, 2021 / Accepted: August 5, 2021 / Published online: August 24, 2021

(C) The Author(s) 2021

\section{ABSTRACT}

Introduction: Published estimates of Alzheimer's disease $(\mathrm{AD})$ progression do not capture the full disease continuum. This study provides transition probabilities of individuals with amyloid- $\beta(\mathrm{A} \beta+)$ pathology across the disease continuum.

Methods: Patient-level longitudinal data from the National Alzheimer's Coordinating Center

Supplementary Information The online version contains supplementary material available at https:// doi.org/10.1007/s40120-021-00272-1.

M. Potashman · M. Levitchi Benea

Biogen, Cambridge, MA, USA

M. Buessing · A. J. Epstein

Medicus Economics, Boston, MA, USA

J. Cummings

Department of Brain Health, Chambers-Grundy Center for Transformative Neuroscience, School of Integrated Health Sciences, UNLV, Las Vegas, NV, USA

S. Borson

University of Washington School of Medicine,

Seattle, WA, USA

S. Borson

The Keck School of Medicine of the University of Southern California, Los Angeles, CA, USA

P. Pemberton-Ross $(\varangle)$

Biogen International GmbH, Neuhofstrasse 30, 6340

Baar, ZG, Switzerland

e-mail: Peter.pembertonross@biogen.com were used to estimate progression rates. Progression rates through five clinically defined $\mathrm{AD}$ stages-asymptomatic, mild cognitive impairment due to $\mathrm{AD}$ (MCI-AD), mild AD dementia, moderate $\mathrm{AD}$ dementia, severe $\mathrm{AD}$ dementiaand death were measured as transition probabilities. Rates were assessed in "incident" patients who recently entered the stage, controlling for covariates. Transition probabilities were generated from multinomial logit regression models that predicted an individual's health state as a function of health state at the previous visit and adjusted for time between initial and follow-up visits, age, sex, years of education, and concomitant symptomatic AD medications.

Results: Annual transition probabilities to more severe dementia stages for surviving incident $\mathrm{A} \beta+$ patients were as follows: asymptomatic to MCI-AD, $40.8 \%$; MCI-AD to mild AD dementia or worse, $21.8 \%$; mild $\mathrm{AD}$ dementia to moderate AD dementia or worse, $35.9 \%$; moderate $\mathrm{AD}$ dementia to severe $\mathrm{AD}$ dementia, $28.6 \%$. Transition probabilities to less severe dementia stages were: $5.3 \%$ annual reversion from MCI-AD to asymptomatic, 3.0\% mild AD dementia to MCI-AD, $1.8 \%$ moderate $\mathrm{AD}$ dementia to mild $\mathrm{AD}$ dementia, and $1.3 \%$ for severe $\mathrm{AD}$ dementia to moderate $\mathrm{AD}$ dementia. Conclusions: These transition probabilities reflect the full continuum of $\mathrm{AD}$ progression in $\mathrm{A} \beta+$ individuals and can be used to assess the impact of treatment on expected transitions. 
Keywords: Alzheimer's disease; Beta amyloid; Dementia; Disease progression; Mild cognitive impairment; Transition probabilities; Economic modeling

\section{Key Summary Points}

\section{Why carry out this study?}

Alzheimer's disease (AD) imposes an enormous economic burden; therefore, valid economic models of disease progression are critical to inform decisionmaking for payers, the healthcare system, and society.

This study used patient-level longitudinal data from the National Alzheimer's Coordinating Center Uniform Data Set to estimate annual $\mathrm{AD}$ progression rates across the five clinically defined $\mathrm{AD}$ stages in individuals with biomarker-confirmed AD pathology.

\section{What was learned from the study?}

Annual transition probabilities to more severe $\mathrm{AD}$ stages were $40.8 \%$

(asymptomatic to mild cognitive impairment due to $\mathrm{AD}$ ), $21.8 \%$ (mild cognitive impairment due to $\mathrm{AD}$ to mild $\mathrm{AD}$ dementia or worse), $35.9 \%$ (mild $\mathrm{AD}$ dementia to moderate $\mathrm{AD}$ dementia or worse), and $28.6 \%$ (moderate $\mathrm{AD}$ dementia to severe $\mathrm{AD}$ dementia).

Calculating transition probabilities using a population of patients with biomarker confirmation of $\mathrm{AD}$, for which the full time spent in the prior stage of disease is accounted for, may increase the precision of estimates.

The estimates derived from this study directly inform the current understanding of $\mathrm{AD}$ progression and can aid in trial design, care planning, and benefit assessments of $\mathrm{AD}$ interventions that reduce progression rates.

\section{INTRODUCTION}

Alzheimer's disease (AD) is a neurodegenerative disorder in which individuals progress clinically from the asymptomatic stages of disease to mild cognitive impairment (MCI) and finally to the mild, moderate, and severe stages of dementia [1-3]. As the most common cause of dementia, AD accounts for approximately $60-80 \%$ of cases and affects $>5$ million patients in the US and approximately 36 million people worldwide $[4,5]$. The Alzheimer's Association estimates that, by 2050, the number of individuals aged $>65$ years with $\mathrm{AD}$ in the US will increase to 13.8 million, and costs of $\mathrm{AD}$ and other dementias will reach $\$ 1.1$ trillion (in 2018 dollars) [4].

Because of the large and growing economic burden of $\mathrm{AD}$, valid economic models of the disease are critical to inform decision-making for payers, the healthcare system, and society. These models require transition probability estimates that accurately reflect progression across the disease continuum. Without such estimates, models assessing the cost-effectiveness of new $\mathrm{AD}$ therapies risk over- or underestimating the true benefits and costs of such interventions.

Multiple estimates of $\mathrm{AD}$ clinical progression rates and transition probabilities across health states have been published, although these vary widely because of differences in data sources, patient sample selection criteria, and analytic methodology [6-11]. For example, estimates of the annual transition probability from severe $\mathrm{AD}$ dementia to death range from 48.0 [7] to $89.8 \%$ [8]. In addition, few studies have attempted to estimate transitions across the full range of disease severity. This is likely due to a paucity of information on relevant indicators of $\mathrm{AD}$ early in the disease, preventing estimates of early transition rates. Furthermore, these estimates often do not account for the duration of time in a particular state, yet the probability of transitioning to a more severe dementia state of $\mathrm{AD}$ likely depends on the amount of time spent in the prior state. To capture the full spectrum of an individual's disease progression, transition probabilities must be examined in "incident" 
patients, individuals who recently entered the stage and for whom the full time spent in the prior stage is known.

The current literature lacks transition probability estimates that account for patient amyloid- $\beta(A \beta)$ status in the brain. $A \beta$ is both a core biomarker of $\mathrm{AD}$ pathophysiology and a major target of disease-modifying therapies [12]. Thus, biomarker confirmation of disease is expected to improve models of disease progression compared with methods based solely on clinical diagnosis of $\mathrm{AD}$ and, in turn, improve clinical trial design and health care system planning. Although previous studies have examined the rate of progression in patients with known amyloid pathology and neurodegeneration, the full spectrum of disease was not captured $[9,10]$. Furthermore, Davis et al. [11] examined transition probabilities across the full spectrum of disease severity; however, no biomarkers were assessed.

To address previous methodological limitations, the current study used patient-level longitudinal data from the National Alzheimer's Coordinating Center (NACC) Uniform Data Set (UDS) [13] to estimate annual progression rates in $A \beta$-bearing $(\mathrm{A} \beta+)$ individuals, using a consistent methodology across the entire $\mathrm{AD}$ continuum.

\section{METHODS}

\section{Data and Sample Selection}

The NACC provided data for this study $[13,14]$. The NACC UDS [13] was used to estimate the transition probabilities associated with $\mathrm{AD}$. This data set contains information on nearly 30,000 patients with varying degrees of cognitive impairment collected from September 2005 through December 2017, from approximately 30 Alzheimer's Disease Research Centers across the US. The NACC conducts quarterly data freezes; therefore, the data used to estimate transition probabilities are specific to the data freeze in December 2017 and the eligibility criteria upon which the data file was created. Data were gathered prospectively by clinicians, neuropsychologists, and other research personnel using standardized protocols and include information on sociodemographic characteristics, dementia history, neurological examination findings, functional status, neuropsychological test results, clinical diagnosis, and apolipoprotein E (ApoE) genotype. In addition, the NACC Neuropathology Data Set from this specific data freeze provides autopsy information for approximately 3000 patients. Because the NACC data were completely deidentified, this study did not qualify as Human Subjects Research under US Department of Health and Human Services Protection of Human Subjects regulations based on guidance from the University of Washington Human Subjects Division. To be included in the sample, patients must have had at least two visits observed in the NACC data. They must also have had either a diagnosis of normal cognition at all visits or a diagnosis of MCI, dementia, or $\mathrm{AD}$ at any visit. Determination of $\mathrm{A} \beta$ status was required (discussed below). The unit of analysis was the patient visit. Patient visit-level observations were recorded in the NACC data on an approximately annual basis. Clinical input and NACC expertise were provided by Drs. Jeffrey Cummings and Soo Borson.

\section{Amyloid Status}

Amyloid status was constructed at the patient level based on information collected during the patient visits. In this study, $A \beta+$ was defined as having any one of the following indicated at a visit: (1) abnormally elevated amyloid on a positron emission tomography scan by visual read, (2) abnormally low amyloid per a cerebrospinal fluid assay, or (3) an autopsy result consistent with a patient having been $\mathrm{A} \beta+$ during their lifetime, defined as frequent neocortical neuritic plaques and a Braak stage of $\mathrm{V}$ or VI. Patients were categorized as $A \beta+$ if they were observed to be $A \beta+$ at any visit within 10 years following their first visit assigned to the asymptomatic/preclinical, $\mathrm{MCI}$ due to $\mathrm{AD}$ (MCI-AD) or mild AD dementia health state. In 
addition, a diagnosis of moderate or severe $\mathrm{AD}$ dementia was considered highly consistent with amyloid positivity by clinical estimation because the likelihood of $A \beta$ positivity at these later stages is $\geq 90 \%$ based on a meta-analysis of numerous studies $[15,16]$. Individuals who were determined to be $A \beta+$ represented $14.8 \%$ of the total population in this NACC data set (a large number of patients in the NACC database have not had amyloid measures of the type used in this study).

\section{Measures}

\section{Health State Definitions}

Visits were assigned to one of six health states listed here in ascending order of disease severity: (1) asymptomatic/preclinical, (2) MCI-AD, (3) mild $\mathrm{AD}$ dementia, (4) moderate $\mathrm{AD}$ dementia, (5) severe $\mathrm{AD}$ dementia, and (6) determined to have died since the last visit. Criteria for the first five health state definitions were based on the $\mathrm{CDR}^{\circledR}$ Dementia Staging Instrument. The Clinical Dementia Rating scale Sum of Boxes (CDR-SB) scores included in the NACC data were used to generate the CDRbased transition probabilities. CDR-SB values for asymptomatic, MCI-AD, mild AD dementia, moderate $\mathrm{AD}$ dementia, and severe $\mathrm{AD}$ dementia were $0,0.5-4.0,4.5-9.0,9.5-15.5$, and 16.0-18.0, respectively [17].

Patient death was captured via an NACC indicator variable (NACCDIED). This variable was derived from the autopsy data and the NACC Milestones form.

\section{Covariates}

Six patient visit-level covariates relevant to transition probabilities were identified in the NACC data, of which five were used in this analysis: (1) time elapsed between current and next visits (measured in years), (2) age at visit (measured in years), (3) patient sex (male, female), (4) years of education, and (5) concomitant symptomatic AD medications identified based on National Institute for Health and
Care Excellence guidance [18] (measured as a binary indicator based on the presence of any of the following medications at any visit: donepezil, galantamine, rivastigmine, memantine).

Additionally, ApoE status (positive vs. negative for the $\varepsilon 4$ allele) was reviewed; specifically, $(\varepsilon 4, \varepsilon 4),(\varepsilon 4, \varepsilon 2)$, and $(\varepsilon 3, \varepsilon 4)$ are the genotypes defined as ApoE $\varepsilon 4$-positive.

\section{Sample Construction}

\section{Construction of Populations Based on Observability of Entry into Health State}

To explore the impact of potential limitations to duration of illness captured within the NACC data, patient visit-level observations were constructed based on whether entry into a health state was observed in the data set. These are referred to as "prevalent" and "incident" populations. The prevalent population did not restrict membership; all observations for patients with at least two visits were included. The incident population included only those in which a patient's new entry into a health state was observed in the data. The incident population is a subset of the prevalent population, so individual patients could contribute visit observations to the calculation of multiple transition probabilities. An illustrative example of visits satisfying the criteria for incident is displayed in Fig. 1. The incident visit population consisted of visits for which an antecedent transition to that visit's health state was observed in the data. For example, a patient's first recorded visit may have been with a health state of MCI-AD, which would not be considered incident because there was no prior visit. If the patient's second visit was for the health state of mild AD dementia, that visit was considered incident, because the patient was observed to have transitioned from MCI-AD to mild AD dementia. The analytic sample was further limited to patient visits for which none of the covariates was missing and an immediately preceding visit was observed. 


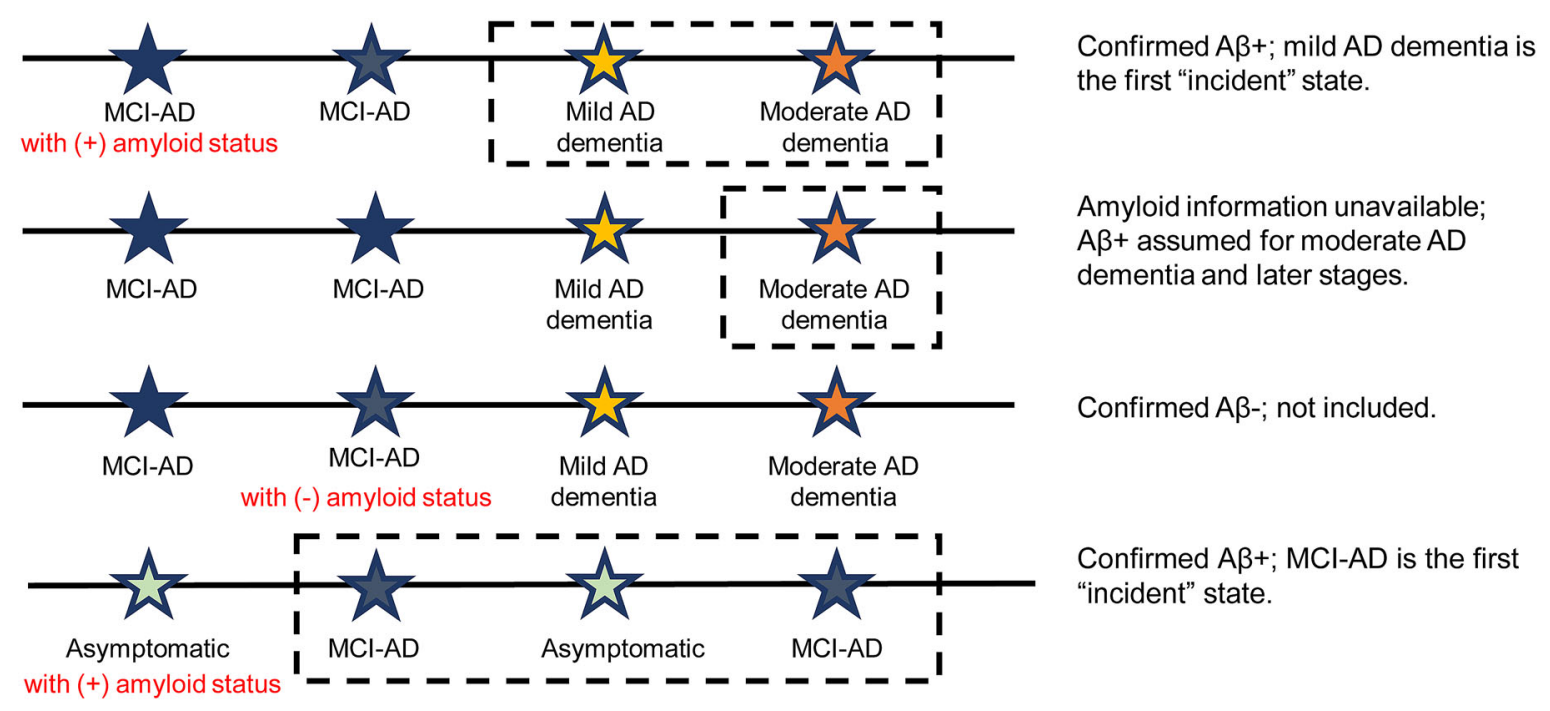

Fig. 1 Incident patient determination. Colors correspond to the labeled stage of disease. Observations that would be included are indicated in the dashed-line boxes.

\section{Analytic Methods}

\section{Descriptive Analyses}

Descriptive analyses were conducted to provide a clinical perspective of the patients in the analytic sample. The main patient characteristics of interest (age, sex, ApoE $\varepsilon 4$, CDR-SB, years of education, concomitant symptomatic $\mathrm{AD}$ medications, and comorbidity profile) were assessed. Measures were summarized with counts and proportions for categorical variables and means and standard deviations for continuous variables.

Prevalence was calculated for the following comorbidities at baseline: diabetes and cardiovascular conditions, including hypertension, hypercholesterolemia, angioplasty/endarterectomy/stent, atrial fibrillation, heart attack/cardiac arrest, cardiac bypass procedure, pacemaker, congestive heart failure, other cardiovascular disease, any cerebrovascular disease, stroke, or transient ischemic attack.

\section{Model Estimation}

The product of these analyses was a set of transition matrices describing the likelihood of moving from each initial AD health state to any other state in 1 year (including remaining in the
$A \beta$ amyloid- $\beta, A D$ Alzheimer's disease, $M C I-A D$ mild cognitive impairment due to Alzheimer's disease

initial state). Each transition matrix was constructed from the data for a specific patient population in which consecutively observed pairs of visits (initial, follow-up) formed a potential state transition. Because the health states were exhaustive, the sum of transition probabilities from any initial state was 1 .

Transition matrices were constructed in three steps. First, patient visit-level data were organized according to initial $\mathrm{AD}$ state (i.e., the state of the first observation in each pair). Next, a full-information maximum likelihood multinomial logit model was developed to predict the outcome state conditional on the initial state. This approach was used to account for censoring from lack of follow-up as well as simultaneous competing risks of transitions to other states. Adjusted models controlled for time between initial and follow-up visits, patient age, sex, and concomitant symptomatic AD medications. ApoE $\varepsilon 4$ was excluded because it was highly collinear with the other predictors in some populations, which led to failures of model convergence. Model estimation produced a transition equation for each pair (initial state-follow-up state) that relates the predictors to the probability of transitioning via the estimated coefficients. In the last step, the transition equations were in turn used to calculate 
mean transition probabilities for each pair (initial state-follow-up state). For $k$ transition states (and the base set to 1), the multinomial logit formula for calculating the probability that individual $j$ transitions to the $i$ th state $\left(p_{i j}\right)$ is

$$
\hat{p}_{i j}=\operatorname{Pr}\left(y_{j}=i\right)=\left\{\begin{array}{ll}
\frac{1}{1+\sum_{m=2}^{k} \exp \left(\mathrm{x}_{j} \beta_{m}\right)}, & \text { if } i=1 \\
\frac{\exp \left(\mathrm{x}_{j} \beta_{i}\right)}{1+\sum_{m=2}^{k} \exp \left(\mathrm{x}_{j} \beta_{m}\right)}, & \text { if } i>1
\end{array},\right.
$$

where $y_{j}$ is individual $j$ 's realized outcome, $x_{j}$ is the row vector of observed values for individual $j$ 's covariates, and $\beta_{m}$ is the coefficient vector from the model predicting transition to state $m$. The simple mean of the predicted probabilities of transitioning to state $i$ is calculated over the $N_{j}$ individuals as

$$
\bar{p}_{i}=\frac{\sum_{n=1}^{N_{j}} \widehat{p}_{i n}}{N_{j}} .
$$

Four covariates were held at constant values for annual transition probability calculations, as follows: (1) time between visits: 365 days; (2) female: 0.5 (representing a 50/50 split in the distribution of patient sex); (3) age: 77 years; (4) years of education: 14 years. The fifth covariate, a binary indicator for whether the patient had concomitant symptomatic AD medications, was allowed to vary; the transition probability for each patient was calculated using that patient's own value for the concomitant medication covariate. Note that covariate values were informed by the data and that the estimated coefficients can generate transition probabilities for any appropriate covariate values.

\section{RESULTS}

\section{Sample Characteristics}

Characteristics of the sample are displayed by incident and prevalent populations (Table 1). The incident population consisted of 3291 unique patients, and the prevalent population comprised 4370 patients. The total numbers of patient visit-level observations were 7027 and 11615 for the incident and prevalent populations, respectively. Tables S1 and S2 in the electronic supplementary materials provide the counts of observed transitions and unique patients by initial health state for the incident and prevalent populations, respectively. Most of the variables assessed showed little difference between these populations, except for CDR-SB health state. In the incident population, the percentages of the asymptomatic, MCI-AD, mild $\mathrm{AD}$ dementia, and moderate $\mathrm{AD}$ dementia patients were generally lower compared with patients in the prevalent population. This result was as expected because the incident population is captured in the prevalent population. Conversely, the percentages of patients in the incident population with severe $\mathrm{AD}$ dementia or those who had died were higher than those observed for patients in the prevalent population.

\section{Transition Probability Estimates}

In this study, annual transition probability estimates were generated for the incident and prevalent populations from a set of transition matrices that modeled the likelihood of moving from each initial AD health state to any other state. Table 2 provides a summary and comparison of the transition estimates across populations after adjustment for time between initial and follow-up visits, patient age, sex, and concomitant symptomatic AD medications. The table shows the percentage of patients in each state who progressed to a more severe dementia state over 12 months. In the incident population, the percentage of asymptomatic patients transitioning to more severe dementia disease states was noticeably higher $(40.8 \%)$ than that observed for the prevalent population $(23.4 \%)$.

The complete adjusted annual transition probabilities for the incident and prevalent populations are shown in Tables 3 and 4, respectively. Annual transition probabilities to more severe dementia stages for surviving patients with known amyloid status were $40.8 \%$ for transitioning from asymptomatic to MCI- 
Table 1 Sample summary statistics among amyloid-restricted patients for both the incident and prevalent populations

\begin{tabular}{|c|c|c|}
\hline Variable & Incident & Prevalent \\
\hline Female, $\%(N)$ & $51.4 \%(3610)$ & $51.0 \%(5923)$ \\
\hline \multicolumn{3}{|l|}{ Age } \\
\hline Mean $(S D)$ & $78.69(10.43)$ & $77.72(10.48)$ \\
\hline Median (IQR) & $80.00(14.00)$ & $79.00(14.00)$ \\
\hline \multicolumn{3}{|l|}{ Years of education } \\
\hline Mean (SD) & $14.84(3.58)$ & $14.73(3.73)$ \\
\hline Median (IQR) & $16.00(6.00)$ & $16.00(6.00)$ \\
\hline Concomitant symptomatic $\mathrm{AD}$ medication use, $\%(N)$ & $64.4 \%(4528)$ & $64.5 \%(7487)$ \\
\hline \multicolumn{3}{|l|}{ ApoE $\varepsilon 4$ genotype, $\%(N)$} \\
\hline$\varepsilon 3, \varepsilon 3$ & $32.5 \%(2285)$ & $32.4 \%(3761)$ \\
\hline$\varepsilon 3, \varepsilon 4$ & $35.6 \%(2505)$ & $36.2 \%(4206)$ \\
\hline$\varepsilon 3, \varepsilon 2$ & $4.6 \%(326)$ & $4.0 \%(462)$ \\
\hline$\varepsilon 4, \varepsilon 4$ & $11.5 \%(806)$ & $11.0 \%(1282)$ \\
\hline$\varepsilon 4, \varepsilon 2$ & $2.4 \%(170)$ & $2.3 \%(266)$ \\
\hline$\varepsilon 2, \varepsilon 2$ & $0.3 \%(20)$ & $0.2 \%(27)$ \\
\hline Missing & $13.0 \%(915)$ & $13.9 \%(1611)$ \\
\hline \multicolumn{3}{|l|}{ CDR health state, $\%(\mathrm{~N})$} \\
\hline Asymptomatic & $0.7 \%(50)$ & $2.9 \%(333)$ \\
\hline MCI-AD & $3.1 \%(219)$ & $9.3 \%(1077)$ \\
\hline Mild AD dementia & $6.3 \%(441)$ & $11.9 \%(1387)$ \\
\hline Moderate $\mathrm{AD}$ dementia & $23.5 \%(1648)$ & $21.5 \%(2495)$ \\
\hline Severe AD dementia & $34.1 \%(2394)$ & $30.6 \%(3553)$ \\
\hline Died & $32.4 \%(2275)$ & $23.8 \%(2770)$ \\
\hline Diabetes & $13.7 \%(752)$ & $13.6 \%(1288)$ \\
\hline CVD risk factors & $78.4 \%(4297)$ & $76.6 \%(7257)$ \\
\hline \multicolumn{3}{|l|}{ Count of CVD components } \\
\hline 0 & $64.7 \%(3547)$ & $66.6 \%(6317)$ \\
\hline 1 & $19.4 \%(1064)$ & $18.7 \%(1774)$ \\
\hline 2 & $9.2 \%(506)$ & $8.8 \%(832)$ \\
\hline 3 & $4.3 \%(235)$ & $3.9 \%(372)$ \\
\hline 4 & $1.5 \%(84)$ & $1.3 \%(125)$ \\
\hline 5 & $0.6 \%(31)$ & $0.5 \%(43)$ \\
\hline 6 & $0.3 \%(14)$ & $0.2 \%(15)$ \\
\hline
\end{tabular}


Table 1 continued

\begin{tabular}{lll}
\hline Variable & Incident & Prevalent \\
\hline Stroke/TIA & $18 \%(972)$ & $16 \%(1560)$ \\
Sample size (observations) & 7027 & 11615 \\
\hline
\end{tabular}

$A D$ Alzheimer's disease, $A p o E$ apolipoprotein E, CDR Clinical Dementia Rating, CVD cardiovascular disease, IQR interquartile range, $M C I-A D$ mild cognitive impairment due to Alzheimer's disease, TIA transient ischemic attack Sample reflects observations used in transition probability models

Table 2 Annual transition probabilities by current health state and sample populations

\begin{tabular}{|c|c|c|c|c|c|}
\hline \multirow[t]{2}{*}{ Sample variant } & \multicolumn{5}{|c|}{$\begin{array}{l}\text { Annual probability of transitioning to a more severe dementia health state from current health } \\
\text { state }\end{array}$} \\
\hline & Asymptomatic & MCI-AD & $\begin{array}{l}\text { Mild AD } \\
\text { dementia }\end{array}$ & $\begin{array}{l}\text { Moderate AD } \\
\text { dementia }\end{array}$ & $\begin{array}{l}\text { Severe AD } \\
\text { dementia }^{a}\end{array}$ \\
\hline Incident population & $40.8 \%$ & $26.5 \%$ & $45.2 \%$ & $59.8 \%$ & $46.7 \%$ \\
\hline Prevalent population & $23.4 \%$ & $36.9 \%$ & $43.4 \%$ & $55.5 \%$ & $41.6 \%$ \\
\hline
\end{tabular}

$A D$ Alzheimer's disease, $M C I-A D$ mild cognitive impairment due to Alzheimer's disease

a Transition from severe AD dementia to death

Table 3 Annual transition probabilities among the incident population with amyloid restriction

\begin{tabular}{lcccccr}
\hline Initial state & \multicolumn{1}{l}{ Next state } & & & \\
\cline { 2 - 6 } & Asymptomatic & MCI-AD & $\begin{array}{l}\text { Mild AD } \\
\text { dementia }\end{array}$ & $\begin{array}{l}\text { Moderate AD } \\
\text { dementia }\end{array}$ & $\begin{array}{l}\text { Severe AD } \\
\text { dementia }\end{array}$ & Died \\
\hline Asymptomatic & $59.2 \%$ & $40.8 \%$ & $0.0 \%$ & $0.0 \%$ & $0.0 \%$ & $0.0 \%$ \\
MCI-AD & $5.3 \%$ & $68.2 \%$ & $15.9 \%$ & $5.7 \%$ & $0.2 \%$ & $4.7 \%$ \\
Mild AD dementia & $0.0 \%$ & $3.0 \%$ & $51.8 \%$ & $31.6 \%$ & $4.3 \%$ & $9.2 \%$ \\
Moderate AD dementia & $0.0 \%$ & $0.0 \%$ & $1.8 \%$ & $38.4 \%$ & $28.6 \%$ & $31.2 \%$ \\
Severe AD dementia & $0.0 \%$ & $0.0 \%$ & $0.0 \%$ & $1.3 \%$ & $52.0 \%$ & $46.7 \%$ \\
\hline
\end{tabular}

$A D$ Alzheimer's disease, $M C I-A D$ mild cognitive impairment due to Alzheimer's disease

Please see Table S1 in the electronic supplementary materials for counts of observed transitions and unique patients by initial state among the incident population with amyloid restriction

$\mathrm{AD}, 21.8 \%$ for MCI-AD to mild AD dementia or worse, $35.9 \%$ for mild AD dementia to moderate $\mathrm{AD}$ dementia or worse, and $28.6 \%$ for moderate $\mathrm{AD}$ dementia to severe $\mathrm{AD}$ dementia (Table 3). Annual transition probabilities to less severe dementia stages were $3.0 \%$ for mild $\mathrm{AD}$ dementia to MCI-AD, $1.8 \%$ for moderate $\mathrm{AD}$ dementia to mild AD dementia, and $1.3 \%$ for severe $\mathrm{AD}$ dementia to moderate $\mathrm{AD}$ dementia (Table 3). Estimated annual transition probabilities in the prevalent population (Table 4) were similar to results from the incident population, although the percentage transitioning from MCI-AD to mild, moderate, or severe $\mathrm{AD}$ 
Table 4 Annual transition probabilities among the prevalent population with amyloid restriction

\begin{tabular}{lcccccr}
\hline Initial state & Next state & & & & & \\
\cline { 2 - 7 } & Asymptomatic & MCI-AD & $\begin{array}{l}\text { Mild AD } \\
\text { dementia }\end{array}$ & $\begin{array}{l}\text { Moderate AD } \\
\text { dementia }\end{array}$ & $\begin{array}{l}\text { Severe AD } \\
\text { dementia }\end{array}$ & Died \\
\hline Asymptomatic & $76.6 \%$ & $21.8 \%$ & $0.7 \%$ & $0.0 \%$ & $0.0 \%$ & $0.9 \%$ \\
MCI-AD & $2.2 \%$ & $60.9 \%$ & $30.7 \%$ & $3.7 \%$ & $0.6 \%$ & $1.9 \%$ \\
Mild AD dementia & $0.0 \%$ & $2.6 \%$ & $54.0 \%$ & $33.6 \%$ & $4.3 \%$ & $5.6 \%$ \\
$\begin{array}{l}\text { Moderate AD } \\
\quad \text { dementia }\end{array}$ & $0.0 \%$ & $0.0 \%$ & $3.0 \%$ & $41.5 \%$ & $30.2 \%$ & $25.3 \%$ \\
Severe AD dementia & $0.0 \%$ & $0.0 \%$ & $0.0 \%$ & $0.9 \%$ & $57.5 \%$ & $41.6 \%$ \\
\hline
\end{tabular}

$A D$ Alzheimer's disease, $M C I-A D$ mild cognitive impairment due to Alzheimer's disease

Please see Table $S 2$ in the electronic supplementary materials for counts of observed transitions and unique patients by initial state among the prevalent population with amyloid restriction

dementia was higher (35.0\% vs. $21.8 \%$; Table 4$)$. Note that some patients progressed by more than one stage in 1 year (e.g., MCI-AD to moderate or severe $\mathrm{AD}$ dementia). The transition probabilities from each stage to death are presented in Tables 3 and 4 .

\section{DISCUSSION}

This study identified transition probabilities across the AD spectrum using a patient population with $A \beta$ pathology in which the entire time spent in the prior disease stage before a transition was known (i.e., incident population). It was hypothesized that transition probabilities, calculated using an $\mathrm{A} \beta+$ confirmed population for which the full time spent in the prior health state was accounted for, would increase the precision of estimates. Analyzed in this study (data not shown) and compared with published transition probabilities in which $A \beta$ status was unknown, transitions observed in $\mathrm{A} \beta+$ individuals were higher in the earlier stages of $\mathrm{AD}$ (i.e., MCI-AD and mild AD dementia) [11]; however, transition probabilities for the moderate AD group align with previously published estimates. One explanation for this observation is that, considering that $\mathrm{AD}$ is the most common cause of dementia [4], most individuals with moderate to severe $\mathrm{AD}$ dementia are expected to have amyloid pathology $[15,16]$. Therefore, the likelihood that our later-stage disease populations would deviate significantly from those previously published is low. Additionally, when using incident population criteria, lower transition estimates were generally observed for the transition from MCI-AD to mild AD dementia and from mild $\mathrm{AD}$ dementia to moderate $\mathrm{AD}$ dementia. Collectively, these results suggest the importance of amyloid confirmation in the earlier stages of $\mathrm{AD}$ (i.e., to distinguish between general MCI vs. MCI due to $\mathrm{AD}$ ) when estimating transition probabilities and that using an incident population can increase the precision of estimates.

Because studies determining transition probabilities are typically conducted with crosssectional data that do not account for patient history of disease (so-called prevalent patients), these estimates may not account for the duration of time spent in the initial state. However, the probability of transitioning to a more severe $\mathrm{AD}$ dementia state is highly dependent on how far the patient has progressed through the prior state when the progression is quantified. Therefore, in this study, transition estimates were calculated in patients who were known to be "incident" to the state in order to capture the full time spent in the disease stage. Restricting the sample to this incident population 
decreased the probability of transition between MCI-AD and mild AD dementia and between mild AD dementia and moderate AD dementia. Transition probabilities calculated using all observations (i.e., the prevalent population) were expected to be biased toward higher transition rates compared with those from the incident population, because patients may have spent unobserved time in their current health state prior to entering the NACC data set. Thus, without observation of the full duration of time spent in the initial state, these patients would appear to be transitioning faster than those who are incident. However, a lower transition probability was not observed in the incident population for the transition from moderate to severe AD dementia. Because the symptoms of moderate to severe $\mathrm{AD}$ dementia are more apparent [4], it may be that patients in these disease stages were diagnosed closer to the time of disease onset and, therefore, most of the time spent in the initial state was captured. Alternatively, lower transition probabilities may not have been uniformly observed in the data because of incomplete sample selection criteria in which the time-in-state (i.e., incident population) hypothesis is only valid for a patient sample different from the one analyzed here, patient heterogeneity, and/or random chance. Additionally, sample sizes were smaller in the early stages of disease due to the determination of $\mathrm{A} \beta+$.

The annual probability of transitioning from an asymptomatic/preclinical stage of disease to MCI-AD in the incident population of this study $(40.8 \%)$ was higher than expected. Because patients in the incident sample were known to be $\mathrm{A} \beta+$, this finding may be a result of this population being biomarker-confirmed for AD pathology and, therefore, not representative of the general asymptomatic population (which may include patients who are not $A \beta+$ ). Furthermore, for individuals in the asymptomatic stage, the definition of incident is not likely to be applicable. Rather, this type of transition would reflect someone on the cusp of an MCI diagnosis or in an early MCI stage. In this context, a prevalent population may be more appropriate for determining transition probabilities in an asymptomatic population.
Although reversion rates were low, transitions to less severe stages were observed. Day-today fluctuations in clinical manifestations of disease may account for this finding. Longer follow-up would be necessary to assess whether these individuals ultimately continued to decline.

\section{Limitations}

Limitations of the data set should be acknowledged. First, although NACC protocols are designed for annual use and longitudinal follow-up, intervals between observations are not precisely 1 year (based on NACC UDS data-collection protocol). Additionally, information on amyloid presence was available only for a small subset of the NACC patient sample (from 2015 onward), and that subset may not be generalizable. In order to maximize the usable sample, a collaboration with clinical experts was undertaken to develop an approach to identify $\mathrm{A} \beta+$ patients based on amyloid testing data that occurred up to 10 years after their first visit in the MCI-AD or mild AD dementia health state. The selection of $A \beta+$ patients via autopsy data may also select for patients who progressed faster and died during the study period.

\section{CONCLUSIONS}

Precise and accurate transition probabilities are critical for economic modeling of $\mathrm{AD}$ and planning for future interventions. However, accurately capturing natural history data in AD presents a host of challenges, especially in the early stages of the disease. When modeling transition probabilities, the precision of clinical staging is imperfect, and the precision of time estimates is limited by the frequency of visits. Examining the entire continuum in one data set produces methodologically consistent estimates across the disease spectrum and is therefore a desirable approach to capturing transition probabilities in an $A \beta+$ sample.

In this study, the NACC UDS sample was used to estimate transition probabilities between $\mathrm{AD}$ health states ranging across the disease continuum in individuals known to be 
$\mathrm{A} \beta+$, from asymptomatic to MCI-AD, mild $\mathrm{AD}$ dementia, moderate $\mathrm{AD}$ dementia, severe $\mathrm{AD}$ dementia, and death. This work directly informs the current understanding of progression along the $\mathrm{AD}$ continuum, and the estimates derived from this study can aid in trial design, care planning, and clinical and economic benefit assessments of $\mathrm{AD}$ interventions that reduce progression rates. The transition probabilities observed in this study, including a low reversion rate, should be further assessed in clinical settings. Furthermore, it will be important to test the consistency of these transition probabilities across countries and over time as the care pathway continues to develop.

\section{ACKNOWLEDGEMENTS}

Funding. This study and the Rapid Service Fee were funded by Biogen (Cambridge, MA, USA). The NACC database is funded by NIA/ National Institutes of Health grant U01 AG016976. NACC data are contributed to by the following NIA-funded Alzheimer's Disease Research Centers: P30 AG019610 (PI Eric Reiman, MD), P30 AG013846 (PI Neil Kowall, MD), P30 AG062428-01 (PI James Leverenz, MD), P50 AG008702 (PI Scott Small, MD), P50 AG025688 (PI Allan Levey, MD, PhD), P50 AG047266 (PI Todd Golde, MD, PhD), P30 AG010133 (PI Andrew Saykin, PsyD), P50 AG005146 (PI Marilyn Albert, PhD), P30 AG062421-01 (PI Bradley Hyman, MD, PhD), P30 AG062422-01 (PI Ronald Petersen, MD, PhD), P50 AG005138 (PI Mary Sano, PhD), P30 AG008051 (PI Thomas Wisniewski, MD), P30 AG013854 (PI Robert Vassar, PhD), P30 AG008017 (PI Jeffrey Kaye, MD), P30 AG010161 (PI David Bennett, MD), P50 AG047366 (PI Victor Henderson, MD, MS), P30 AG010129 (PI Charles DeCarli, MD), P50 AG016573 (PI Frank LaFerla, PhD), P30 AG062429-01 (PI James Brewer, MD, PhD), P50 AG023501 (PI Bruce Miller, MD), P30 AG035982 (PI Russell Swerdlow, MD), P30 AG028383 (PI Linda Van Eldik, PhD), P30 AG053760 (PI Henry Paulson, MD, PhD), P30 AG010124 (PI John Trojanowski, MD,
PhD), P50 AG005133 (PI Oscar Lopez, MD), P50 AG005142 (PI Helena Chui, MD), P30 AG012300 (PI Roger Rosenberg, MD), P30 AG049638 (PI Suzanne Craft, PhD), P50 AG005136 (PI Thomas Grabowski, MD), P30 AG062715-01 (PI Sanjay Asthana, MD, FRCP), P50 AG005681 (PI John Morris, MD), and P50 AG047270 (PI Stephen Strittmatter, MD, PhD).

Medical Writing and Other Assistance. Research assistance was provided by Julia Bond from Medicus Economics and was funded by Biogen. Medical writing support, under direction of the authors, was provided by Katie Partrick, Ph.D. at MediTech Media and was funded by Biogen.

Authorship. All named authors meet the International Committee of Medical Journal Editors (ICMJE) criteria for authorship for this article, take responsibility for the integrity of the work as a whole, and have given their approval for this version to be published.

Authors' Contributions. Marric Buessing, Jeffrey Cummings, and Andrew J. Epstein contributed to the study conception and design. Material preparation, data collection, and analysis were performed by Marric Buessing and Andrew J. Epstein. All authors commented on previous versions of the manuscript. All authors read and approved the final manuscript.

Prior Presentation. Part of this manuscript is based on work that was previously presented as a poster (P070) at the virtual Clinical Trials in Alzheimer's Disease (CTAD) 2020 congress held on November 4-7, 2020.

Disclosures. Soo Borson reports no conflicts of interest. Michele Potashman was an employee of Biogen at the time of this study. Mihaela Levitchi Benea and Peter PembertonRoss are employees and shareholders of Biogen. Marric Buessing and Andrew J. Epstein are employees of Medicus Economics, which received funding from Biogen to participate in this research. Jeffrey Cummings has provided consultation to Acadia, Actinogen, Alkahest, Alzheon, AriBio, Avanir, Axsome, Behren 
Therapeutics, Biogen, Cassava, Cerecin, Cortexyme, EIP Pharma, Eisai, Foresight, GemVax, Genentech, Green Valley, Grifols, Janssen, Karuna, Merck, Novo Nordisk, Otsuka, ReMYND, Resverlogix, Roche, Samumed, Samus, Signant Health, Sunovion, Suven, and United Neuroscience pharmaceutical and assessment companies. He has stock options in ADAMAS, Alzheon, AnnovisBio, MedAvante, and BiOasis; owns the copyright of the Neuropsychiatric Inventory; and is supported by NIGMS grant P20GM109025, NINDS grant U01NS093334, National Institute on Aging (NIA) grant R01AG053798, NIA grant P20AG068053, and NIA grant R35AG71476.

Compliance with Ethics Guidelines. The NACC provided data for this study [13, 14]. Because the NACC data were completely deidentified, this study did not qualify as Human Subjects Research under US Department of Health and Human Services Protection of Human Subjects regulations based on guidance from the University of Washington Human Subjects Division.

Data Availability. The data sets analyzed during the current study are not publicly available from the authors due to compliance with the NACC Data Use Agreement. The NACC data are publicly available from the NACC via their data request process: https://naccdata.org/ requesting-data/data-request-process.

Open Access. This article is licensed under a Creative Commons Attribution-NonCommercial 4.0 International License, which permits any non-commercial use, sharing, adaptation, distribution, and reproduction in any medium or format, as long as you give appropriate credit to the original author(s) and the source, provide a link to the Creative Commons licence, and indicate if changes were made. The images or other third party material in this article are included in the article's Creative Commons licence, unless indicated otherwise in a credit line to the material. If material is not included in the article's Creative Commons licence and your intended use is not permitted by statutory regulation or exceeds the permitted use, you will need to obtain permission directly from the copyright holder. To view a copy of this licence, visit http://creativecommons.org/licenses/by$\mathrm{nc} / 4.0 /$.

\section{REFERENCES}

1. Sperling RA, Aisen PS, Beckett LA, et al. Toward defining the preclinical stages of Alzheimer's disease: recommendations from the National Institute on Aging-Alzheimer's Association workgroups on diagnostic guidelines for Alzheimer's disease. Alzheimers Dement. 2011;7:280-92.

2. Albert MS, DeKosky ST, Dickson D, et al. The diagnosis of mild cognitive impairment due to Alzheimer's disease: recommendations from the National Institute on Aging-Alzheimer's Association workgroups on diagnostic guidelines for Alzheimer's disease. Alzheimers Dement. 2011;7:270-9.

3. McKhann G, Knopman DS, Chertkow H, et al. The diagnosis of dementia due to Alzheimer's disease: recommendations from the National Institute on Aging-Alzheimer's Association workgroups on diagnostic guidelines for Alzheimer's disease. Alzheimers Dement. 2011;7:263-9.

4. Alzheimer's Association. 2020. Alzheimer's disease facts and figures. https://www.alz.org/media/ Documents/alzheimers-facts-and-figures.pdf. Last updated March 10, 2020. Accessed 19 Jan 2021.

5. World Health Organization. Dementia cases set to triple by 2050 but still largely ignored. https://www. who.int/mediacentre/news/releases/2012/ dementia_20120411/en/. Last updated April 11, 2012. Accessed 19 Jan 2021.

6. Liu D, Zhou X-H. Covariate adjustment in estimating the area under ROC curve with partially missing gold standard. Biometrics. 2013;69:91-100.

7. Spackman E, Kadiyala S, Neumann PJ, Veenstra DL, Sullivan SD. Measuring Alzheimer disease progression with transition probabilities: estimates from NACC-UDS. Curr Alzheimer Res. 2012;9:1050-8.

8. Bloudek LM, Spackman DE, Veenstra DL, Sullivan SD. CDR state transition probabilities in Alzheimer's disease with and without cholinesterase inhibitor intervention in an observational cohort. J Alzheimers Dis. 2011;24:599-607.

9. Iaccarino L, Sala A, Perani D, Alzheimer's Disease Neuroimaging Initiative. Predicting long-term clinical stability in amyloid-positive subjects by FDG-PET. Ann Clin Transl Neurol. 2019;6:1113-20. 
10. Vos SJ, Verhey F, Frölich L, et al. Prevalence and prognosis of Alzheimer's disease at the mild cognitive impairment stage. Brain. 2015;138:1327-38.

11. Davis M, O'Connell T, Johnson S, et al. Estimating Alzheimer's disease progression rates from normal cognition through mild cognitive impairment and stages of dementia. Curr Alzheimer Res. 2018;15: 777-88.

12. Jack CR Jr, Bennett DA, Blennow K, et al. NIA-AA research framework: toward a biological definition of Alzheimer's disease. Alzheimers Dement. 2018;14:535-62.

13. Besser L, Kukull W, Knopman DS, et al. Version 3 of the National Alzheimer's Coordinating Center's Uniform Data Set. Alzheimer Dis Assoc Disord. 2018;32:351-8.

14. National Alzheimer's Coordinating Center. Webbased query system. https://naccdata.org. Accessed 19 Jan 2021.
15. Ossenkoppele R, Jansen WJ, Rabinovici GD, et al. Prevalence of amyloid PET positivity in dementia syndromes: a meta-analysis. JAMA. 2015;313: 1939-50.

16. Ossenkoppele R, Rabinovici GD, Smith R, et al. Discriminative accuracy of [18F]flortaucipir positron emission tomography for Alzheimer disease vs other neurodegenerative disorders. JAMA. 2018;320:1151-62.

17. O'Bryant SE, Lacritz LH, Hall J, et al. Validation of the new interpretive guidelines for the clinical dementia rating scale sum of boxes score in the national Alzheimer's coordinating center database. Arch Neurol. 2010;67:746-9.

18. National Institute for Health and Care Excellence. Donepezil, galantamine, rivastigmine and memantine for the treatment of Alzheimer's disease. NICE guidance TA217. https://www.nice.org.uk/ guidance/ta217/chapter/3-The-technologies. Last updated March 23, 2011. Accessed 19 Jan 2021. 